\title{
Energetics of $n$-Alkanes in Zeolites: A Configurational-Bias Monte Carlo Investigation into Pore Size Dependence
}

\author{
Simon P. Bates, ${ }^{*}, \dagger$ Willy J. M. van Well, ${ }^{\dagger}$ Rutger A. van Santen, ${ }^{\dagger}$ and Berend Smit ${ }^{\ddagger}$ \\ Contribution from the Schuit Institute of Catalysis, Laboratory for Inorganic \\ Chemistry \& Catalysis, Eindhoven University of Technology, P.O. Box 513, \\ 5600 MB Eindhoven, The Netherlands, and Shell International Oil Products, B.V., \\ Shell Research and Technology Centre, Amsterdam, P.O. Box 38000, \\ 1030 BN Amsterdam, The Netherlands
}

Received November 17, 1995. Revised Manuscript Received May 8, $1996^{\otimes}$

\begin{abstract}
A recently-developed Monte Carlo method is used to simulate the energetics of $n$-alkanes from butane to decane in a variety of different all-silica zeolite structures (MFI, MOR, FAU, RHO, LTA, and FER). Where possible, the predicted values of the heat of adsorption are compared to experimental data and are generally found to be in good agreement. On the basis of the energetic data, the graphs of heat of adsorption as a function of mean pore diameter appear to show a maximum between 4 and $5 \AA$. However, close inspection of the location and conformation of the alkanes in small pore zeolites reveals that the molecules adopt highly coiled conformations localized exclusively in regions of maximum void volume. In the case of the small pore zeolites studied here-RHO and LTA-these maximum void volumes are the $\alpha$-cages and the alkanes "feel" a larger pore diameter than that generally used to characterize the zeolite (that of the channels). It is necessary to obtain information on the location and conformations of sorbed molecules to fully understand the trends in the heat of adsorption as a function of pore diameter.
\end{abstract}

\section{Introduction}

Zeolites are highly crystalline inorganic materials composed of corner-sharing $\mathrm{AlO}_{4}$ and $\mathrm{SiO}_{4}$ tetrahedra. The linking of these tetrahedra to form extended three-dimensional structures naturally gives rise to a series of windows, channels, or cages within the zeolite. This porosity means the ratio of internal to external surface area is very high. It is this fact, together with the high thermal stability and possibility of acid sites within the pores, that make zeolites such a valuable class of catalytic materials for use in the petrochemical industry. ${ }^{1}$

For a great many of the catalytic applications of zeolites, a clear picture of the nature and location of adsorbed molecules is fundamental to a mechanistic understanding of catalytic processes. Experimental studies such as $\mathrm{NMR}^{2,3}$ can provide information on the location ${ }^{2}$ and diffusion ${ }^{3}$ of small sorbates, e.g. Xe. The energetics of alkane sorption in zeolites have been studied experimentally for nearly 30 years. As early as 1966 Barrer ${ }^{4}$ demonstrated the additivity of dispersion energies for hydrocarbon sorbates in NaX zeolite. However, it is difficult to obtain accurate information for larger, catalytically-interesting molecules. Longer chain alkanes are one such example of molecules for which comparatively little experimental information is available. The energetics of adsorption as a function of pore diameter have also been investigated experimentally; Stach et al. ${ }^{5}$ have shown that the heat of adsorption of butane decreases logarithmically as the pore diameter is increased from that of

* Address correspondence to this author

Eindhoven University of Technology.

$¥$ Shell Research and Technology Centre.

${ }^{\otimes}$ Abstract published in Advance ACS Abstracts, June 15, 1996.

(1) Naber, J. E.; de Jong, K. P.; Stork, W. H. J.; Kuipers, H. P. C. E.; Post, M. F. M. Studies in Surface Science \& Catalysis. Zeolites and Related Microporous Materials: State of The Art 1994; Weitkamp, J., Karge, H. G., Pfeifer, H., Hölderich, W., Eds.; Elsevier: Amsterdam, 1994.

(2) Chmelka, B. F.; Raferty, D.; McCormick, A. V.; de Menorval, L. C.; Levine, R. D.; Pines, A. Phys. Rev. Lett. 1991, 66, 580-583.

(3) Kärger, J.; Ruthven, D. M. Diffusion in Zeolites and other Microporous Solids; Wiley \& Sons: New York, 1992.

(4) Barrer, R. M. J. Colloid Interface Sci. 1966, 21, 415-434. silicalite. Such a decrease in the heat of adsorption as the pore diameter increases is widely accepted to be true; however, a systematic study of sorption in smaller pore zeolites has not been performed.

Thus, this is an area ideally suited to the application of theoretical simulations to determine the behavior and properties of sorbates in zeolites. The recent advances in computer hardware, together with the rapid development of commercial, user-friendly software, ${ }^{6}$ have made these types of calculations accessible to more and more researchers. A collection of review articles from $1992^{7}$ demonstrates just how much progress has been made in this area in recent years.

Previous simulations, based on molecular dynamics calculations, ${ }^{8,9}$ Monte Carlo calculations, ${ }^{10}$ or a hybrid Monte Carlo and energy minimization procedure, ${ }^{11}$ have tended to concentrate on small guest molecules. The reason for this is invariably one of available CPU resources; the larger the guest molecule, the more demanding the calculation. The Configurational-Bias Monte Carlo method (CB-MC) ${ }^{12,13}$ has recently been developed for chain molecules and avoids these limitations. As in conventional Monte Carlo methods, the technique has the advantage of not having to follow the "natural path" of adsorbent molecules through the host. Instead, it is possible to move a guest to an arbitrary point in the host structure. The CB-MC

(5) Stach, H.; Jänchen, J.; Thamm, H.; Stiebitz, E.; Vetter, R. A. Ads. Sci. Technol. 1986, 3, 261-270.

(6) Biosym Technologies/Molecular Simulations Inc.: 9865 Scranton Road, San Diego, CA.

(7) Catlow, C. R. A., Ed. Modelling of Structure and Reactivity in Zeolites; Academic Press: London, 1992.

(8) June, R. L.; Bell, A. T.; Theodorou, D. N. J. Phys. Chem. 1992, 96, 1051-1060.

(9) Dumont, D.; Bougeard, D. Zeolites 1995, 15, 650-655.

(10) Goodbody, S. J.; Watanabe, K.; MacGowan, D.; Walton, J. P. R. B.; Quirke, N. J. Chem. Soc., Faraday Trans. 1991, 87 (13), 1951-1958. (11) Freeman, C. M.; Catlow, C. R. A.; Thomas, J. M.; Brode, S. Chem. Phys. Lett. 1991, $186(2,3), 137$.

(12) Smit, B.; Siepmann, J. I. J. Phys. Chem. 1994, 98 (34), 84428452.

(13) Smit, B. Mol. Phys. 1995, 85 (1), 153-172. 
Table 1. Details of Zeolite Simulation Boxes

\begin{tabular}{llccccc}
\hline \multicolumn{1}{c}{ zeolite } & code & no. of cells ${ }^{a}$ & no. of O & $\begin{array}{c}\text { av pore } \\
\text { diameter }^{b}\end{array}$ & $a^{b}$ & $b^{b}$ \\
\hline silicalite $^{c}$ & MFI & $2 \times 2 \times 4$ & 3072 & $5.4^{d}$ & 40.140 & 39.840 \\
mordenite $^{c}$ & MOR & $2 \times 2 \times 4$ & 1536 & 6.7 & 36.388 & 40.940 \\
faujasite & FAU & $2 \times 2 \times 2$ & 3072 & 7.4 & 48.515 & 48.515 \\
zeolite RHO & RHO & $3 \times 3 \times 3$ & 2592 & 3.6 & 45.093 & 45.093 \\
zeolite A & LTA & $2 \times 2 \times 2$ & 3072 & 4.1 & 49.220 & 49.220 \\
ferrierite & FER & $2 \times 3 \times 6$ & 2592 & $4.8^{d}$ & 38.312 & 42.3824 \\
\end{tabular}

${ }^{a}$ Number of cells, $k \times l \times m$, in the $a, b$, and $c$ directions, respectively. ${ }^{b}$ Box dimensions and pore diameters in $\AA$. ${ }^{c}$ Data taken from ref 12 . ${ }^{d}$ Pore diameters for MFI [010] and FER [001] given; see text of Section 4.

differs from conventional Monte Carlo in so far as the guest species is grown atom by atom inside the zeolite, rather than inserted as a complete molecule. The rapidly-decreasing probability of accepting an insertion as chain length increases, inherent in conventional Monte Carlo methods, is therefore absent. The method is more fully described elsewhere ${ }^{12,13}$ and has already been successfully applied to study the sorption of alkanes in silicalite and mordenite ${ }^{12,14}$ and adsorption isotherms in silicalite. ${ }^{13,15,16}$

In this paper, we report CB-MC calculations of the sorption energetics of $n$-alkanes from butane to decane in several allsilica zeolites. The energetics of alkane sorption are correlated with the average zeolite pore diameter. The question of alkane location or siting is a complementary study that is to be addressed in detail in a future publication. ${ }^{17}$ It is worth noting here that "siting" or "location" can mean different things for different zeolites. In the case of a zeolite with only one type of channel, e.g. zeolite RHO, it refers to the location and conformation of the alkane with respect to the channels. However, in the case of a zeolite with more than one type of channel, e.g. silicalite, it means not only the location of the alkane in a channel but also the distribution over the different types of channels.

In the next section, details are given of the models used for alkanes and zeolites in the calculations. Section 3 contains information pertaining to the energetics of the $n$-alkanes in different zeolites. Section 4 contains a discussion of the energetics as a function of pore diameter and Section 5 the conclusions that may be drawn from this study.

\section{Computational Models and Details}

The computational models for the alkanes and zeolites are similar to those used previously in CB-MC simulations ${ }^{12-16}$ and are summarized briefly in this section. The $\mathrm{CH}_{3}$ and $\mathrm{CH}_{2}$ groups of the alkanes are treated as single interaction centers-the so-called "united atom" approach. These interaction centers are separated by a fixed bond length of $1.54 \AA$. Bond bending between three interaction centers is modeled by a simple harmonic potential. ${ }^{18}$ Energy as a function of torsion angle between four interaction centers is given by a Jorgenson potential. ${ }^{19}$ Interaction centers in the same molecule that are more than three bonds apart interact according to a 12-6 Lennard-Jones potential. The values for the Lennard-Jones size and energy parameters, and indeed all parameters that describe the intra-alkane potential terms, are identical to those used in a previous CB-MC study of alkane sorption in silicalite. ${ }^{12}$ Using these parameters, the vapour-liquid phase equilibria of $\mathrm{C}_{8}$ to $\mathrm{C}_{48}$ alkanes are accurately described over a large temperature range. ${ }^{20,21}$

(14) Smit, B.; Siepmann, J. I. Science 1994, 264, 1118-1120.

(15) Smit, B. J Phys. Chem. 1995, 99 (15), 5597-5603.

(16) Smit, B.; Maesen, T. L. Nature 1995, 374, 42-44.

(17) Bates, S. P.; van Well, W. J. M.; van Santen, R. A.; Smit, B. J. Phys. Chem. Submitted for publication.

(18) van der Ploeg, P.; Berendsen, H. J. C. J. Chem. Phys. 1982, 76, $3271-3276$.

(19) Jorgensen, W. L.; Madura, J. D.; Svenson, C. J. J. Am. Chem. Soc. 1984, 106, 6638-6646.
The zeolite is assumed to be a rigid structure, in accordance with the work of Kiselev et al. ${ }^{22}$ The potential at a series of grid points is initially calculated and then the potential at any point in the zeolite can be determined during the simulations by interpolation. ${ }^{23}$ In all calculations, a grid spacing no larger than $0.18 \AA$ was used. Grids of this spacing have been shown to vastly increase computational efficiency, while still preserving the accuracy of the potential to within $0.4 \%{ }^{12}$ The intermolecular potential between zeolite and alkane is assumed to be dominated by terms from the zeolite oxygen atoms; the potential contributions from the silicon atoms are implicit in the oxygen atom parameters. The interaction between zeolite $\mathrm{O}$ atoms and the $\mathrm{CH}_{n}$ groups of the alkane is represented by a 12-6 Lennard-Jones potential, the energy and size parameters of which are given in ref 12 and are almost identical to those used in a molecular dynamics simulation by June et al. ${ }^{8}$ The potential is truncated at $13.8 \AA$ and the usual tail corrections are applied.

Table 1 gives details of the different zeolite simulation boxes that were used in the calculations. In all cases the simulation boxes had all three angles equal to $90^{\circ}$. Periodic boundary conditions were imposed on all our calculations. The zeolite structures were normally taken from the crystallographic information in the Solids Builder Module of the BIOSYM Catalysis and Sorption Project software. ${ }^{6}$ (Notable exceptions are faujasite and RHO, for reasons described below.) This database of zeolite structures generally contains the crystallographic data for the as-synthesized aluminosilicate structure: for our calculations we used MFI $,{ }^{24} \mathrm{MOR},{ }^{25} \mathrm{RHO},{ }^{26} \mathrm{LTA},{ }^{27}$ and FER.${ }^{28}$ In all these cases, all aluminium atoms are replaced with silicon. This does introduce an uncertainty because the structure of the all-silica end member is unlikely to be the same as that of the aluminosilicate. However, it is believed that this uncertainty is small in most cases, smaller than other inherent approximations such as the rigidity of the zeolite. Exceptions to this general rule are faujasite and RHO. For faujasite we use the purely siliceous structure determined by Cheetham et al..$^{29}$ on the basis of a neutron diffraction and MAS-NMR study. The cell parameter of the all-silica structure is predicted to be almost $1 \AA$ smaller than the database aluminosilicate structure. ${ }^{6}$ This large difference in lattice parameter (and concomitant contraction in pore diameter by $0.2 \AA$ ) is likely to have a significant effect on predicted sorption energetics. In the case of zeolite RHO, we use the database crystal structure ${ }^{26}$ and in addition perform a lattice energy minimization

(20) Smit, B.; Karaborni, S.; Siepmann, J. I. J. Chem. Phys. 1995, 102 (5), 2126-2140. 332 .

(22) Bezus, A. G.; Kiselev, A. V.; Lopatkin, A. A.; Du, P. Q. J. Chem. Soc., Faraday Trans. 2 1978, 74, 367-379.

(23) June, R. L.; Bell, A. T.; Theodorou, D. N. J. Phys. Chem. 1990, 94, $1508-1516$.

(24) van Koningsveld, H.; van Bekkum, H.; Jansen, J. C. Acta Crystallogr. 1987, B43, 127-132.

(25) Alberti, A.; Davoli, P.; Vezzalini, G. Z. Kristallogr. 1986, 175, 249256.

(26) McCusker, L. B.; Baerlocher, C. New Developments in Zeolites Science and Technology; Proceedings of the 6th International Zeolite Conference; Olsen, D., Bisio, A., Eds., Butterworth: Guildford, UK, 1984; pp $812-822$

(27) Gramlich, V.; Meier, W. M. Z. Kristallogr., Kristallgeom., Kristallphys., Kristallchem., 1971, 133, 134-149.

(28) Vaughan, P. A. Acta Crystallogr. 1966, 21, 983-990.

(29) Hriljac, J. A.; Eddy, M. M.; Cheetham, A. K.; Donohue, J. A.; Ray, G. J. J. Solid State Chem. 1993, 106, 66-72. 
on the all-silica structure, as the framework is known to undergo marked distortion upon dehydration. ${ }^{30,31}$

The simulations of the sorption of each alkane in each different zeolite are carried out in a number of cycles. Each cycle consists of a randomly selected attempt to either translate a molecule, to rotate it, to regrow part of it, or to regrow all of it. The latter two steps exploit the efficiency of the CB-MC technique. The maximum values for rotation and displacement are chosen so that $50 \%$ of these moves are accepted. The number of accepted regrows was always greater than 1000 in a simulation run, ensuring a reliable prediction of the Henry coefficient which is calculated from data collected during the regrow step. (The Henry coefficient may be thought of as a thermodynamic measure of the ease of adsorption of the molecules in the zeolite.) In order to satisfy this constraint, a typical simulation consists of at least $10^{6}$ total cycles. The efficiency of the CB-MC method is such that equilibrium is normally reached within 100 cycles. The entire simulation of decane in silicalite took approximately 21 CPU hours on a Silicon Graphics workstation equipped with an R4400 processor. The values of the Henry coefficient are not presented in this paper. They do, however, exhibit similar trends as a function of chain length as the heats of adsorption. (In the limit of low coverage, the heat of adsorption and the Henry coefficient are related according to eq 19 in ref 12.)

The calculation of the heat of adsorption was performed at infinite dilution, i.e. one alkane molecule. The calculation of the heat of adsorption requires a simulation of the alkane in the ideal gas state, as a reference point. These data, collected for a single alkane in an infinitely large simulation box, are identical to that in ref 12 . The heat of adsorption is calculated according to the equations given in ref 12 . All simulations were performed at a temperature of $298 \mathrm{~K}$.

\section{Energetics of $\boldsymbol{n}$-Alkane Sorption}

In this section, we report the heats of adsorption of $\mathrm{C}_{4}$ to $\mathrm{C}_{10}$ alkanes in a variety of different all-silica zeolites. The data for silicalite and mordenite have been published previously ${ }^{12}$ and so only brief comments are made regarding these results. We report data for the following zeolites: FAU, RHO, LTA, and FER.

(a) Silicalite and Mordenite. The energetics of $n$-alkane sorption in silicalite and mordenite are included here primarily for the discussion on the behavior of the heat of adsorption as a function of pore diameter. In addition, we make a comparison between the mordenite calculations and experimental studies. ${ }^{32-35}$

It has already been demonstrated in ref 12 that the CB-MC technique predicts heats of adsorption of alkanes in silicalite in very good agreement with the wealth of experimental data available. For low carbon-number alkanes, the theoretical CB$\mathrm{MC}$ values are fractionally lower than the experimental values. This trend is reversed for nonane and higher alkanes. The theoretical data give a slope of $11.0 \mathrm{~kJ} / \mathrm{mol}$ per carbon atom for $\mathrm{C}_{4}$ to $\mathrm{C}_{8}$ and $12.9 \mathrm{~kJ} / \mathrm{mol}$ per carbon atom for $\mathrm{C}_{8}$ to $\mathrm{C}_{12}$.

The simulated sorption in mordenite predicts consistently lower values of the heat of adsorption than for silicalite. This is in accordance with the larger average pore diameter of mordenite, resulting in a smaller dispersive interaction with the zeolite. The slope of the graph of heat of adsorption versus carbon number is $9.3 \mathrm{~kJ} / \mathrm{mol}$ per carbon atom. Far less experimental literature data are available for mordenite; we have found two experimental sorption energies of $n$-hexane and a

(30) McCusker, L. B. Zeolites 1984, 4, 51-55.

(31) Parise, J. B.; Gier, T. E.; Corbin, D. R.; Cox, D. E. J. Phys. Chem. 1984, $88,1635-1640$.

(32) Derouane, E. G.; Nagy, J. B.; Fernandez, C.; Gabelic, Z.; Laurent, E.; Maljean, P. Appl. Catal. 1988, 40, L1-L10.

(33) Jänchen, J.; Jahn, E.; Lohnse, U.; Stach, H. Preprints of the 3rd German Workshop on Zeolite Chemistry; Berlin-Dahlem: Berlin, 1991; p 16.

(34) Stach, H.; Fiedler, K.; Jänchen, J. Pure Appl. Chem. 1993, 65 (10), 2193-2200.

(35) Eder, F.; Stockenhuber, M.; Lercher, J. A. Stud. Surf. Sci. Catal. 1995, $97,465-500$.

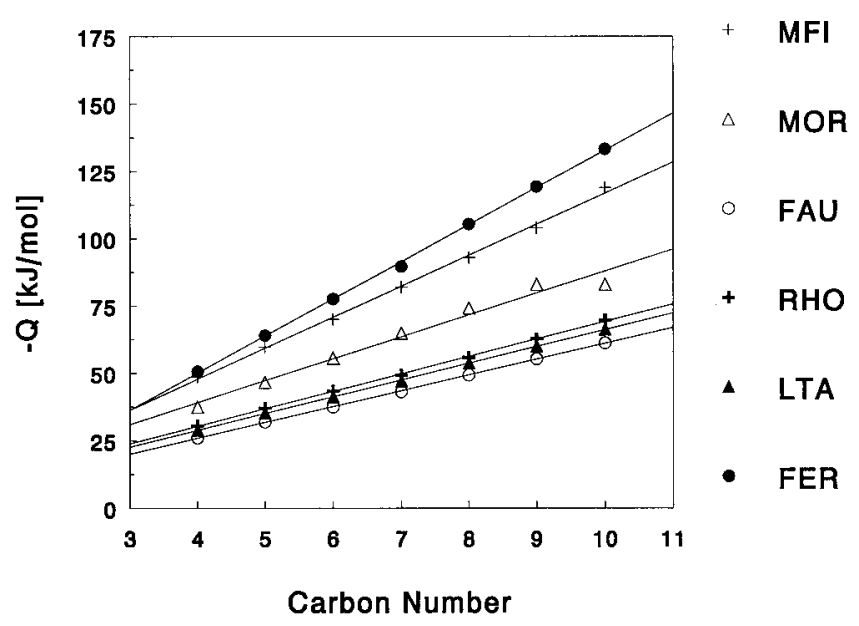

Figure 1. Heat of adsorption $(Q)$ of $n$-alkanes in zeolites as a function of carbon number (lines of best fit are drawn through data).

series of values for $\mathrm{C}_{3}$ to $\mathrm{C}_{6}$. The first value for hexane ${ }^{32}$ is $-68 \mathrm{~kJ} / \mathrm{mol}$, determined at $363 \mathrm{~K}$ for a sample of unknown composition. The second ${ }^{33,34}$ is $-59.0 \mathrm{~kJ} / \mathrm{mol}$, measured at 298 $\mathrm{K}$ on a virtually aluminium-free mordenite sample. The latter of these two values is more directly comparable to the value from CB-MC simulations in ref 12 and is found to be only 3 $\mathrm{kJ} / \mathrm{mol}$ larger. This discrepancy is well within the range of experimental values determined for alkane sorption in silicalite. ${ }^{12}$ Eder et al. ${ }^{35}$ have recently reported sorption values for $\mathrm{C}_{3}$ to $\mathrm{C}_{6}$ ( -41 to $-69 \mathrm{~kJ} / \mathrm{mol}$, respectively) on a MOR sample with a $\mathrm{Si} / \mathrm{Al}$ ratio of 10 . From these results the incremental heat of adsorption per carbon atom is calculated as $9.3 \mathrm{~kJ} / \mathrm{mol}$, identical to that predicted by our simulations. While the presence of aluminium appears to increase the heat of adsorption, the incremental value per carbon atom appears to be relatively unaffected. This is commented on further in the faujasite results.

The temperature at which the first literature value ${ }^{32}$ was measured $(363 \mathrm{~K})$ is believed to have a small influence on the difference between experimental and theoretical values. Recent calculations we have performed for the sorption of alkanes in silicalite suggest that the heat of adsorption decreases with increasing temperature, though very slowly (approximately $10^{-2}$ $\mathrm{kJ} / \mathrm{mol}$ per deg $\mathrm{K}$ for hexane). This near-independence of the heat of adsorption with increasing temperature for alkanes shorter than $\mathrm{C}_{8}$ has also been reported by Maginn et al. ${ }^{36}$ for a similar CB-MC study of alkane sorption in silicalite.

(b) Faujasite. The calculated heats of adsorption of $\mathrm{C}_{4}$ to $\mathrm{C}_{10}$ alkanes in all-silica faugasite are given in Table 2. Also included in the table are experimental values for a sample of US-Ex. ${ }^{5}$ Figure 1 includes a graph of the heat of adsorption as a function of carbon number for sorption in FAU.

In these simulations, the all-silica faujasite structure determined by Cheetham et al. ${ }^{29}$ was used, as has been stated previously. The reason for this is that there appeared to be a significant difference between the all-silica structure and the original (aluminosilicate) crystal structure, ${ }^{37}$ large enough to affect the predicted heats of adsorption. In supplementary calculations (data not presented here) using the aluminosilicate library structure, we have found this to be true. Heats of adsorption of $n$-alkanes in the all-silica FAU with the aluminosilicate structure were all less negative than the values obtained for FAU with an all-silica structure. In the case of $\mathrm{C}_{6}$, the deviation was $12 \%$ of the all-silica value.

(36) Maginn, E. J.; Bell, A. T.; Theodorou, D. N. J. Phys. Chem. 1995, 99, 2057-2079.

(37) Olson, D. H. J. Phys. Chem. 1970, 74, 2758-2764. 
Table 2. Heat of Adsorption, $Q$, Values for Alkanes in Several All-Silica Zeolites

\begin{tabular}{|c|c|c|}
\hline zeolite code & alkane chain length & $Q[\mathrm{~kJ} / \mathrm{mol}]$ \\
\hline \multirow[t]{7}{*}{ FAU } & $\mathrm{C}_{4}$ & $-26.0(-34.6)^{b}$ \\
\hline & $\mathrm{C}_{5}$ & $-31.9(-41.0)$ \\
\hline & $\mathrm{C}_{6}$ & $-37.5(-46.7)$ \\
\hline & $\mathrm{C}_{7}$ & -43.1 \\
\hline & $\mathrm{C}_{8}$ & $-49.3(-56.3)$ \\
\hline & $\mathrm{C}_{9}$ & -55.3 \\
\hline & $\mathrm{C}_{10}$ & $-61.2(-67.1)$ \\
\hline \multirow[t]{7}{*}{ RHO } & $\mathrm{C}_{4}$ & -30.5 \\
\hline & $\mathrm{C}_{5}$ & -37.1 \\
\hline & $\mathrm{C}_{6}$ & -43.3 \\
\hline & $\mathrm{C}_{7}$ & -49.3 \\
\hline & $\mathrm{C}_{8}$ & -55.8 \\
\hline & $\mathrm{C}_{9}$ & -62.7 \\
\hline & $\mathrm{C}_{10}$ & -69.7 \\
\hline \multirow[t]{7}{*}{ distorted $\mathrm{RHO}^{a}$} & $\mathrm{C}_{4}$ & -32.3 \\
\hline & $\mathrm{C}_{5}$ & -39.3 \\
\hline & $\mathrm{C}_{6}$ & -46.1 \\
\hline & $\mathrm{C}_{7}$ & -52.6 \\
\hline & $\mathrm{C}_{8}$ & -59.6 \\
\hline & $\mathrm{C}_{9}$ & -66.8 \\
\hline & $\mathrm{C}_{10}$ & -74.4 \\
\hline \multirow[t]{7}{*}{ LTA } & $\mathrm{C}_{4}$ & $(-36.6)^{c}-29.0$ \\
\hline & $\mathrm{C}_{5}$ & -35.2 \\
\hline & $\mathrm{C}_{6}$ & -41.2 \\
\hline & $\mathrm{C}_{7}$ & -47.1 \\
\hline & $\mathrm{C}_{8}$ & -53.7 \\
\hline & $\mathrm{C}_{9}$ & -60.0 \\
\hline & $\mathrm{C}_{10}$ & -66.3 \\
\hline \multirow[t]{7}{*}{ FER } & $\mathrm{C}_{4}$ & -50.7 \\
\hline & $\mathrm{C}_{5}$ & -64.0 \\
\hline & $\mathrm{C}_{6}$ & -77.6 \\
\hline & $\mathrm{C}_{7}$ & -89.7 \\
\hline & $\mathrm{C}_{8}$ & -105.4 \\
\hline & $\mathrm{C}_{9}$ & -119.3 \\
\hline & $\mathrm{C}_{10}$ & -133.3 \\
\hline
\end{tabular}

${ }^{a}$ See text for explanation of "distorted RHO". ${ }^{b}$ Experimental values in parentheses, from ref $5 .{ }^{c}$ See text for explanation of value in parentheses.

The calculated heats of adsorption for alkanes in faujasite shown in Table 2 are significantly less than those found for alkane sorption in both silicalite and mordenite, ${ }^{12}$ in agreement with the larger pore size of faujasite. The values are somewhat low in comparison with experimental data obtained at $300 \mathrm{~K}$ on a sample of US-Ex: ${ }^{5}$ an ultrastable extracted form of an almost totally dealuminated Y zeolite. The sample used in this study had a $\mathrm{Si} / \mathrm{Al}$ ratio of $95 .{ }^{38}$ In sorption experiments it was found to contain "a limited number of adsorption sites of high energy". The presence of framework or extra-framework aluminium is a possible explanation for the difference between experimental and theoretical results. For a more meaningful assessment of the accuracy of the theoretical results, a range of experimental data should ideally be examined. This is clearly shown by the calculations of alkane sorption in silicalite; ${ }^{12}$ a range of experimental values, sometimes differing by up to 10 $\mathrm{kJ} / \mathrm{mol}$ for the same alkane, were examined and "selected" values were used in comparison. Such a range of experimental measurements is not available for all-silica faujasites. There is, of course, always the possibility that the model potential used in the calculation may be inaccurate. However, the present results for all the zeolites studied do not suggest that it is necessary to change the model.

Although the actual values of the predicted heat of adsorption are lower than experimental data, the theoretical and experimental values of the heat of adsorption per carbon atom are in good agreement: the simulations predict a slope of $5.8 \mathrm{~kJ} / \mathrm{mol}$

(38) Stach, H.; Lohse, U.; Thamm, H.; Schirmer, W. Zeolites 1986, 6 $74-90$. per carbon, while the experimental data $^{34}$ yield a value of 6.0 $\mathrm{kJ} / \mathrm{mol}$ per carbon. Once again, it appears that the aluminium content in the experimental sample has a greater effect on the absolute values of the sorption energy than the incremental value per carbon atom. This may be rationalized by considering a zeolite with a small amount of aluminium in the framework. For low $\mathrm{Al}$ concentrations, it is reasonable to assume that only one carbon atom of the alkane interacts with the aluminium site. The remainder of the alkane will see a local environment that is Al-free. Thus, a low concentration of Al will cause a shift of the heat of adsorption of the individual alkanes, but the slope of the heat of adsorption as a function of carbon number will remain the same.

(c) Zeolite RHO. The simulated heats of adsorption of alkanes in zeolite RHO are shown in Table 2 and the results are plotted graphically on Figure 1. It can be seen that the heat of adsorption values are larger than those predicted for faujasite, yet smaller than those for silicalite and mordenite. ${ }^{12}$

Zeolite RHO belongs to the cubic crystal class. Its framework topology consists of truncated cubo-octahedrons ( $\alpha$-cages) linked via double-eight rings. ${ }^{26}$ This generates a pore structure that consists of two equivalent, interpenetrating 3-dimensional circular channel systems of $3.6 \AA$ diameter. As a comparison to the data shown in Table 2, the heat of adsorption of butane through decane varies from 49 to $119 \mathrm{~kJ} / \mathrm{mol}$ for alkanes sorbed in silicalite. ${ }^{12}$ Thus, we predict a decrease in the heat of adsorption as the average pore diameter of the zeolite decreases from approximately 5.5 to $3.6 \AA$.

A literature value is available for the sorption of $n$-butane in zeolite RHO. Barrer and Rosemblat ${ }^{39}$ measured a heat of adsorption of $45 \mathrm{~kJ} / \mathrm{mol}$ in a sample of RHO with a $\mathrm{Si} / \mathrm{Al}$ ratio of approximately 6. Our predicted value in all-silica RHO is $31 \mathrm{~kJ} / \mathrm{mol}$. It is most probable that the presence of aluminium is responsible for the difference. (It has been found that the heats of adsorption of $n$-pentane and $n$-hexane in ZSM5 -silicalite with framework aluminium-are increased by some $10 \mathrm{~kJ} / \mathrm{mol}$ compared to silicalite. ${ }^{40}$ )

Zeolite RHO is known to undergo an appreciable distortion and loss of symmetry upon dehydration. ${ }^{30,31}$ It is reasonable to speculate on the stability of the all-silica form of such a malleable framework. To test the stability we performed a series of supplementary calculations. Firstly, the all-silica structure was subjected to a lattice energy minimization using the METAPOCS code, ${ }^{41}$ implemented in the BIOSYM Catalysis and Sorption Software Suite. ${ }^{6}$ Then, the minimized periodic structure was used in a further series of CB-MC alkane sorption calculations. The METAPOCS minimization included Buckingham, three-body, and shell-model terms and the calculation was performed in $P 1$ symmetry. Following the simulation, the symmetry of the minimized structure was recovered and found to be $\operatorname{Im} 3 m$, identical to that of the original crystal structure. A computation of IR stretching frequencies predicted no imaginary frequencies, indicating the minimized structure to be a true minimum rather than a saddle point.

The minimized structure shows a decrease in cell parameter from 15.03 to $14.78 \AA$. The pores remain circular and actually increase in diameter slightly, by $0.2 \AA$. This minimization shows that the all-silica form of zeolite RHO does indeed appear

(39) Barrer, R. M.; Rosemblat, M. A. New Developments in Zeolite Science and Technology; Proceedings of the 6th International Zeolite Conference; Olsen, D., Bisio, A., Eds.; Butterworths: Guildford, UK, 1984; pp 276-287.

(40) van Well, W. J. M.; Wolthuizen, J. P.; van Hooff, J. H. C. Unpublished results, 1995. UK. 
to be stable and has a framework geometry that is only slightly different from that assumed in the previous calculations.

We then used the minimized unit cell of RHO as the starting point for a further series of CB-MC calculations. The results are reported in Table 2, under the heating of "distorted RHO". (These values are not plotted on Figure 1 for the sake of clarity.) It can be seen from the data that the heats of adsorption in the distorted RHO are slightly larger than for alkane sorption in the undistorted zeolite. This is in accordance with the slight increase in pore diameter upon distortion. The heats of adsorption in distorted RHO are between 2 (butane) and $5 \mathrm{~kJ} /$ mol (decane) larger.

(d) Zeolite A. Zeolite A (LTA) ${ }^{27}$ is another cubic zeolite with a similar framework structure to RHO. Whereas the RHO structure consists of $\alpha$-cages joined through double-eight rings, zeolite $\mathrm{A}$ is formed by joining the $\alpha$-cages through single eight rings. Whereas the body-center of the RHO unit cell contains an $\alpha$-cage, the center of the LTA cell contains a $\beta$-cage. The pores of zeolite A are approximately $0.5 \AA$ larger than those of RHO (4.1 $\AA$ ). The inclusion of this zeolite in our simulations is mainly to reinforce the predictions from the RHO calculations.

The simulated values of the heats of adsorption are shown in Table 2, and the values are presented graphically on Figure 1. The slope of the graph of heat of adsorption versus carbon number is calculated to be approximately $6.1 \mathrm{~kJ} / \mathrm{mol}$ per carbon, slightly lower than that of zeolite RHO (approximately $6.3 \mathrm{~kJ} /$ mol per carbon).

The calculations show a result for butane that at first sight seems somewhat anomalous, but is in fact easily explained once the location of the sorbed molecules is considered. Butane appears to have a higher heat of adsorption than pentane in zeolite A, contrary to what is intuitively expected. It transpires that during the course of the simulations, many of the butane molecules are inserted into and then grown inside the $\beta$-cages of the structure. These are smaller than the $\alpha$-cages, resulting in a greater dispersive interaction between the alkane and the zeolite wall, thus leading to a higher heat of adsorption. The heat of adsorption is shown in parentheses In Table 2.

Experimentally, it is highly unlikely that butane molecules can migrate through the six-ring windows of the $\beta$-cage. Thus, for our calculations to accurately simulate the sorption of butane, the molecules should be discouraged from growing inside these regions of pore volume. This is achieved by placing a single extra oxygen atom at the center of every $\beta$-cage. This has a negligible effect on the heats of adsorption of alkanes that are larger than butane; the heats of adsorption of pentane and hexane are increased by less than $0.3 \mathrm{~kJ} / \mathrm{mol}$ using this method. More importantly, an analysis of locations shows that the butane molecules populate the $\alpha$-cages exclusively. This value of the heat of adsorption for butane in zeolite $\mathrm{A}$ is given without parentheses in Table 2.

(e) Ferrierite. Ferrierite ${ }^{28}$ is a zeolite with two straight, elliptical channels; a 10-ring channel along [001], $5.4 \times 4.2 \AA$, and an 8-ring channel along [010], $4.8 \times 3.5 \AA$. Data from the simulations of $\mathrm{C}_{4}$ to $\mathrm{C}_{10}$ alkanes in ferrierite are shown in Table 2 and presented graphically on Figure 1. The values of the heat of adsorption are larger than for any other zeolite considered. The slope of the line of best fit on Figure 1 is calculated as $13.5 \mathrm{~kJ} / \mathrm{mol}$ per carbon atom. It should be stressed that the present calculations do not consider the distribution of the alkane molecules over the channel systems in ferrierite; this is considered elsewhere in a separate study. ${ }^{17}$

Little experimental data are available for comparison with our theoretical heats of adsorption. Derouane et al. ${ }^{32}$ quote a value of $87.2 \mathrm{~kJ} / \mathrm{mol}$ for the sorption of $n$-hexane in a ferrierite

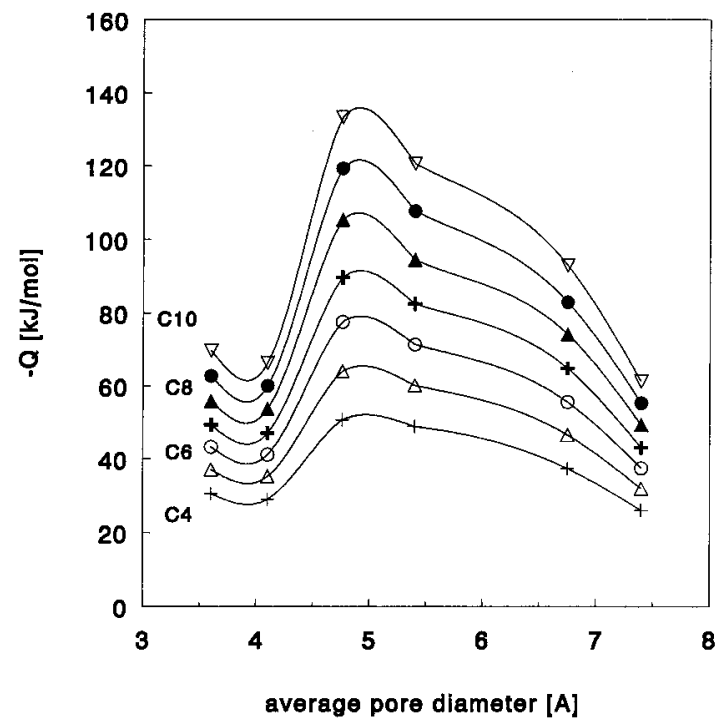

Figure 2. Heat of adsorption $(Q)$ of $n$-alkanes in zeolites as a function of mean pore diameter. (A spline is fitted to the data as a guide to the eye.)

sample of unknown composition at $363 \mathrm{~K}$. This is to be compared with the value of $78 \mathrm{~kJ} / \mathrm{mol}$ given in Table 2 . While there are a number of factors which prevent direct comparisone.g. compositional and temperature differences-the familiar difference of approximately $10 \mathrm{~kJ} / \mathrm{mol}$ does reinforce the integrity of the calculations.

For the sake of completeness, it is worth mentioning a recent diffraction, NMR, and computational study of the purely siliceous form of ferrierite. ${ }^{42}$ In this work, the all-silica framework is refined in the space group Pmnn instead of the Immm group usually attributed to aluminosilicate ferrierite frameworks. The study predicts subtle changes in the structure of the all-silica end member; lattice energy minimization predicts the Pmnn form to be a mere $0.5 \mathrm{~kJ} / \mathrm{mol}$ lower in energy than the Immm form. The present calculations use the Immm structure for the ferrierite lattice. It seems unlikely that the minimization of an all-silica FER framework and subsequent use in the CB-MC calculations would yield heats of adsorption significantly different from those described earlier.

\section{Alkane Adsorption as a Function of Pore Diameter}

From the calculations of alkane sorption in various zeolites reported in the previous section, it is possible to collate the data to illustrate how the sorption depends on the average zeolite pore diameter. Figure 2 shows this graphically.

The pore diameters plotted on Figure 2 are the sizes of the channel openings that are generally used to characterize the various different zeolites. In addition, average pore diameter is applicable to those zeolites with non-circular pore systems. In these cases, the average pore diameter is simply calculated as the arithmetic mean of the pore dimensions. Zeolites with channels of different dimensions or topology along different crystallographic axes have more than one (average) pore diameter. Examples of such zeolites in the present calculation series are MFI and FER. In the case of MFI, the distribution of alkanes in the channels has already been investigated by CBMC calculations. ${ }^{12}$ However, the small difference in average pore diameter between the straight and sinusoidal channels makes it somewhat arbitrary which effective pore diameter is used. We use the average pore diameter of the straight channel

(42) Morris, R. E.; Weigel, S. J.; Henson, N. J.; Bull, L. M.; Janicke, M. T.; Chmelka, B. F.; Cheetham, A. K. J. Am. Chem. Soc. 1994, 116, 11849-11855. 


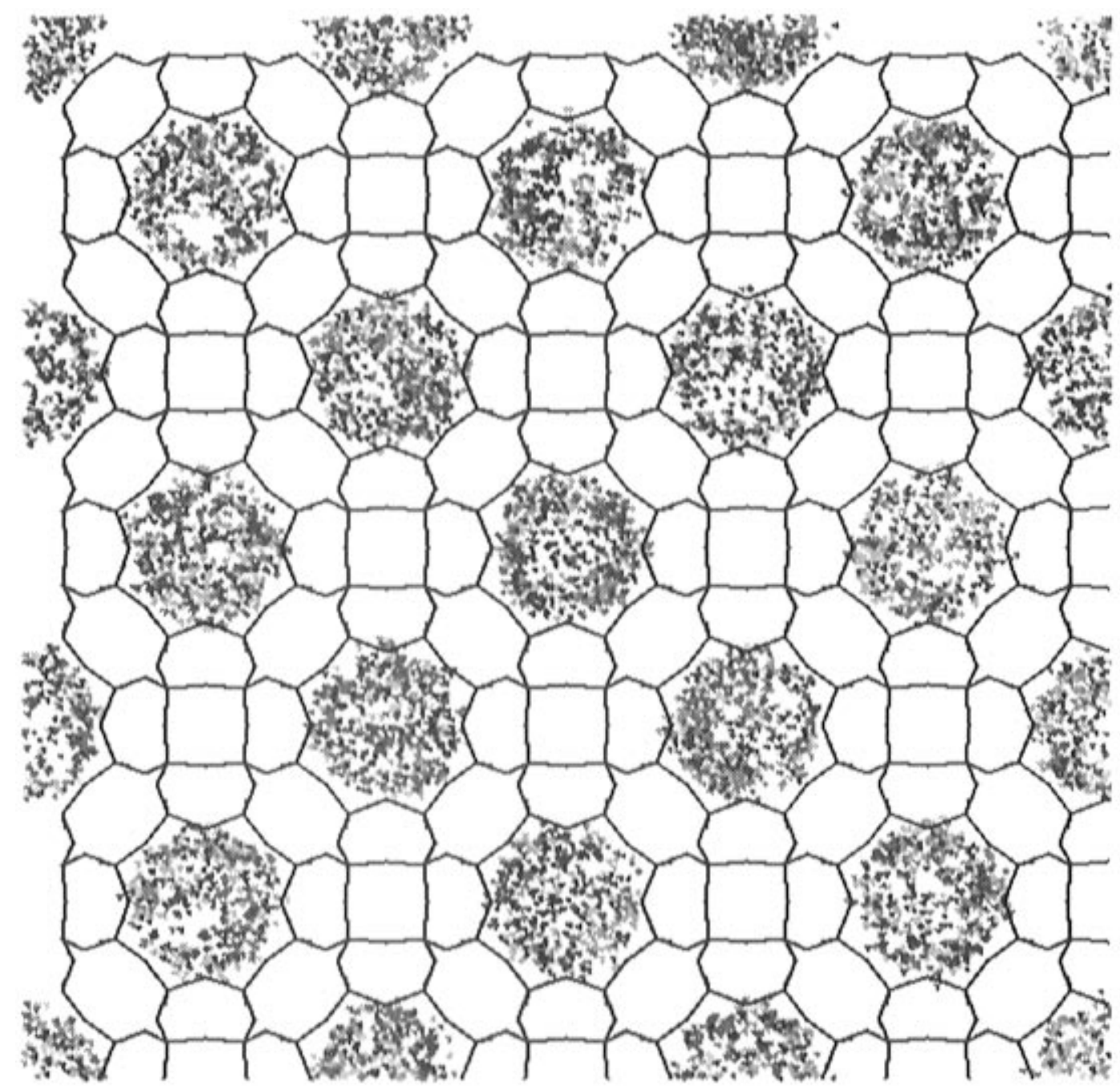

Figure 3. Locations of the center of hexane molecules adsorbed in zeolite RHO. (Viewed down [100], each dot represents the midpoint of the central $\mathrm{C}-\mathrm{C}$ bond in hexane.)

here, i.e. $5.4 \AA$. In the case of FER, a zeolite with two straight channels of mean pore diameter $4.8 \AA$ down [001] and $4.1 \AA$ down [010], we take the mean pore diameter of $4.8 \AA$ for this system. (An analysis of location of sorbed molecules shows this channel to be exclusively populated for pentane and longer alkanes. ${ }^{17}$ )

Figure 2 shows that the optimum adsorption of alkanes in all-silica zeolites is exhibited by a zeolite with an average pore diameter between 4 and $5 \AA$. The heat of adsorption steadily increases as the pore diameter decreases from $7.4 \AA$ to approximately $5 \AA$. At pore diameters smaller than this value, the heat of adsorption is predicted to decrease. For zeolites FAU and LTA, nearly identical heats of adsorption are predicted although their pore diameters and topologies are completely different. From the figure, it would appear that ferrierite is the ideal zeolite with which to maximize the heat of adsorption of a straight-chain alkane.

It is reasonable to assume that this behavior of adsorption energy as a function of pore size arises primarily due to greater Lennard-Jones intermolecular repulsion as the zeolite pore size decreases. For zeolites with a pore diameter less than that which gives the maximum heat of adsorption on Figure 2, the smaller distances between alkane and zeolite lead to a less favorable intermolecular potential, and thus a lowered heat of adsorption. This explains the decrease in heat of adsorption going from FER to LTA, but not the small increase seen when going from LTA to RHO.
These predictions echo the findings of Derycke et al. ${ }^{43}$ for physisorption in a confined geometry, who note that adsorption energy increases with decreasing pore diameter and repulsive effects play a more important role for smaller zeolites. However, the situation is somewhat more complex and this becomes apparent upon examining the conformation and distribution of alkanes sorbed in zeolites RHO and LTA.

Irrespective of chain length, the sorbed alkanes studied in this work are all found in the center of the $\alpha$-cages of RHO-the largest available void volume. By contrast, alkanes in other zeolites such as silicalite visit all parts of the pore structure during the simulations. ${ }^{12}$ Figure 3 illustrates the confinement of the sorbed molecules to the $\alpha$-cages in RHO; each of the dots represents the center of the central bond of a hexane molecule. It is clear from this figure that the center point of all alkanes lies at or near the center of the $\alpha$-cage. This location for sorption seems always to be more favorable than locating the center point of any hexane molecules in the double-eight rings that connect the cages. A plot of the end $\mathrm{CH}_{3}$ groups rather than the midpoints produces a similar picture.

This total preference for sorption in the $\alpha$-cages results in alkane chains that are highly coiled. To illustrate this point we compare the decane molecule in RHO and in the larger of the two channels in FER. In RHO, approximately $70 \%$ of the decane chains contain four or more gauche defects. By

(43) Derycke, I.; Vigneron, J. P.; Lambin, P.; Lucas, A. A.; Derouane, E. G. J. Chem. Phys. 1991, 94 (6), 4620-4627. 
comparison, over $90 \%$ of decane molecules sorbed in the 10 ring of FER have an all-trans conformation. The fact that the alkanes in RHO are so tightly coiled means that the whole alkane molecule is confined to the $\alpha$-cage. Not even in the case of decane do the tail carbon atoms extend into the eight rings that connect the cages.

A similar picture is seen for zeolite LTA, though the effect is less extreme due to the structure of zeolite A ( $\alpha$-cages linked by single-eight rings). Once again, with the exception of butane as discussed in the previous section, sorption is confined to the centers of the $\alpha$-cages and the longer alkanes adopt a highly coiled conformation. We have performed supplementary calculations on other structures (e.g. the all-silica version of the AlPO AWW-an effectively unidimensional material with a pore diameter of $3.9 \AA$ ). We do not present the data here, but similar features to the conformation of alkanes in RHO and LTA are seen. It appears that this highly coiled preferential sorption in the largest possible void volume is a general feature of smallpore materials. Ferrierite is the smallest pore material we have found that does not show this preferential sorption. In ferrierite, hexane and higher alkanes are evenly distributed throughout the 10-ring channel (4.8 $\AA$ mean pore diameter).

The present simulations do not consider the migration of the alkanes into their favored positions for sorption. In the cases of zeolites RHO and A, migration must occur through the eightring windows, even though these are not preferred sites for sorption. There is evidence from both experimental and theoretical studies to suggest that diffusion of alkanes through the eight-ring windows of RHO and A is feasible; a negative (i.e. favorable) heat of adsorption for butane in RHO has been experimentally measured by Barrer and Rosemblat. ${ }^{39}$ In addition, a molecular dynamics investigation of methane diffusion into dealuminated zeolite A by Demontis and Suffritti ${ }^{44}$ has shown migration through the eight-rings is possible.

The preferential siting in the $\alpha$-cages has important consequences for the graphs presented in Figure 2. The "average pore diameter" that alkanes in RHO and LTA feel is in fact the diameter of the $\alpha$-cage. This has been estimated for the $\alpha$-cages of RHO and LTA as between 10.4 and 11.6 $\AA^{45}$ Using the Characterise module of the Biosym Catalysis and Sorption Project Software, ${ }^{6}$ we estimate the maximum diameters of the $\alpha$-cages of RHO and LTA to be 10.4 and $11.5 \AA$, respectively. These values are all located far to the right of the maximum on the graphs. The fractionally higher heats of adsorption in RHO can be explained on the grounds of increased dispersive interaction between zeolite and alkane (as the $\alpha$-cages in RHO are slightly smaller than those in LTA). We can therefore conclude that on the basis of the sorption energy data alone, the shape of graphs shown on Figure 2 is true. Only when the location and conformation of alkanes is examined do we see that characterization of the zeolites RHO and LTA on Figure 2 by their usual pore diameters gives too simplistic a view. The average pore diameter for these materials is larger than the pore diameters that are commonly used to characterize them, as a

(44) Demontis, P.; Suffritti, G. B. Studies in Surface Science \& Catalysis. Zeolites and Related Microporous Materials: State of The Art 1994; Weitkamp, J., Karge, H. G., Pfeifer, H., Hölderich, W., Eds.; Elsevier: Amsterdam, 1994; pp 2107-2113.

(45) Abrams, L.; Corbin, D. R. J. Inclusion Phenom. Mol. Recognit. Chem. 1995, 21, 1-46. result of the location of the alkanes. We then predict a simpler inverse relationship between the heat of adsorption and the average pore diameter-the larger the pore diameter the lower the heat of adsorption.

Figures 1 and 2 show that topologically different zeolites may exhibit similar overall sorption energies for a given alkane; the structures of LTA and FAU are quite different, yet the heats of adsorption are very nearly equal. In addition, from an analysis of conformation and location, we see that sorption in LTA and FAU induces different conformations in sorbed alkanes. The catalytic function of these zeolite materials is a complex property, dependent on many factors including the diffusion of guest species, as well as their location, conformation, and sorption energy. The CB-MC method provides valuable information on a number of these factors.

\section{Conclusions}

In this paper, we have presented a series of configurationalbias Monte Carlo calculations concerning the adsorption energetics of $n$-alkanes in a variety of different zeolites. The following conclusions may be drawn from the study:

(i) In all the systems we have studied, there is a strong thermodynamic driving force for sorption, even in zeolites with pore diameters smaller than $4 \AA$. For several zeolites we have been unable to find sufficient experimental data to critically assess the performance of the simulations. Where comparisons can be made, we have found the predicted energy values to be generally in good agreement with those from literature.

(ii) For all the zeolites we have studied, the predicted heat of adsorption was found to increase linearly with alkane chain length. The heat of adsorption per carbon atom varies from $5.8 \mathrm{~kJ} / \mathrm{mol}$ per carbon for FAU to $13.5 \mathrm{~kJ} / \mathrm{mol}$ per carbon for FER.

(iii) In order to obtain a true picture of the variation of the heat of adsorption as a function of pore diameter, it is necessary to look at the location and conformation of the sorbed species as well as the energetic data. Based on energetic data alone, the graph of the heat of adsorption as a function of mean zeolite pore diameter exhibits a maximum between 4 and $5 \AA$. However, when the location and conformation of the sorbed species is examined, a simpler inverse relationship between pore diameter and heat of adsorption is found. The reason for this is that alkanes in smaller-pore materials preferentially absorb in a localized, highly coiled state in the largest void volume in the structure, rather than distributed throughout the structure. These alkanes therefore "feel" a larger pore diameter than that which is generally used to characterize the material.

The need to obtain information on the conformation and location of sorbed species has been clearly demonstrated and the CB-MC method has been shown to be very useful in providing this. In the future we plan to use this technique to more fully examine the location and conformation of the alkanes in the different zeolites. ${ }^{17}$

Acknowledgment. The authors wish to express their gratitude to Florian Eder for pointing out an inconsistency in an earlier version of the manuscript and to Dr. David Corbin for useful discussions.

JA953856Q 